\title{
Two-dimensional convection-diffusion problem solved using method of localized particular solutions
}

\author{
Juraj Mužík ${ }^{1, *}$ and Martina Holičková ${ }^{1}$ \\ ${ }^{1}$ University of Zilina, Faculty of Civil Engineering - Department of geotechnics, Univerzitná 8215/1, \\ Slovakia
}

\begin{abstract}
A meshless local method of approximated particular solutions (LMAPS) is used to analyze problem described by the convectiondiffusion equation. The method solves the steady convection-diffusion equation with reaction term. The discretized system of equations is derived via interpolation procedure using integrated multiquadrics (MQ) radial basis functions (RBF). The solution of the equation is performed over simple geometry with non-uniform velocity field and results are presented in the article. The LMAPS method is capable to produce stable solutions with results comparable to the analytical solutions.
\end{abstract}

\section{Introduction}

Meshless approaches of approximated particular solution are receiving increased attention due to their accuracy associated with the special type of RBF function used and the flexibility they offer as the meshing requirements are either eliminated or reduced.

The main drawback of mesh-based numerical methods is the requirement of the mesh generation, which may be difficult to process during the numerical implementations, especially for multi-dimensional problems. In order to avoid mesh generation process many meshless numerical methods, such as the local boundary integral element method (LBIEM) [1], smoothed particle hydrodynamics (SPH) method [2], meshless strong (collocation) method [3] and meshless local Petrov-Galerkin method (MLPG) [4] have been developed.

The local method of approximated particular solution (LMAPS) was proposed by Cheng et al. and was applied to elliptic problems [5] and non-linear problems [6]. In LMAPS the domain is covered by cloud of scattered nodes. In the work on LMAPS reported so far, the support of the any computational node is taken to be a simple subdomain in a shape of a circle though in theory the domain can be of any shape, with the computational node in the centre of the circle. The most often used interpolation for field variables were the moving least-squares, though Sellountos and Sequeira [7] used augmented thin plate spline (ATPS) radial basis functions (RBFs) for interpolation of the field variable and gradients over the circular boundaries.

The LMAPS formulation used in this article represents a stable, accurate solution tool. Numerical experiment of the quasi two-dimensional linear convection-diffusion-reaction

*Corresponding author: muzik@,fstav.uniza.sk 
equation solution is presented in the article with the comparison against the analytical solution, which verifies the performance of the LMAPS.

\section{Localized method of approximated particular solutions}

In order to apply the LMAPS, the following procedure may be adopted to solve physical variable $u$ with any given differential operator. Even though the implementation details of the LMAPS are quite similar to the meshless collocation methods, its origin follows the globally defined method of approximated particular solution (MAPS) (see [8]). In this study the LMAPS is treated as special case of the meshless collocation method with the support domain concept already included in the formulation.

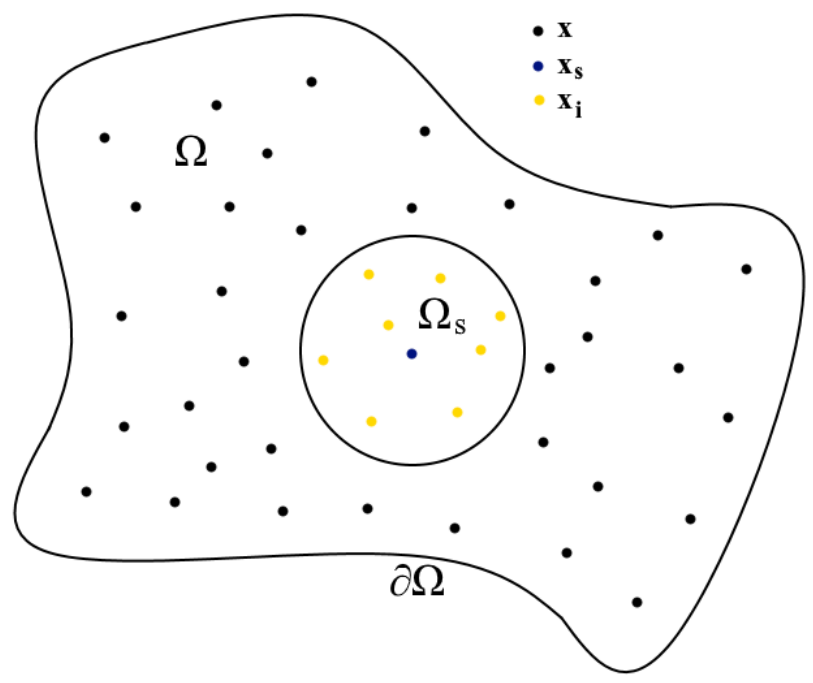

Fig. 1. The diagram of global domain $\Omega$, local support domain $\Omega_{s}$ of point $x_{s}$, global points $x$ and local point $x_{i}$.

The area of interest $\Omega$ with the boundary $\partial \Omega$ is covered by points within the area and also on the global boundary (see Fig.1). Consider a local circular (or any simple shape e.g. rectangle) sub-domain $\Omega_{s}$ centered at every point $s$. This sub-domain is called support domain and using the points in a particular support domain any function can be expressed using just nodal values as

$$
u(\mathbf{x})=\sum_{i=1}^{N S} \alpha_{i} F\left(\left\|\mathbf{x}-\mathbf{x}_{i}\right\|\right), x \in \Omega_{s}
$$

where $N S$ is the total number of computational nodes in the support domain, $F$ is the radial basis function, $\mathbf{x}$ is coordinate vector and $\alpha_{i}$ are the local weighting coefficients to be determined. Because the values of $u$ expressed using (1) at the computational nodes should be the same as $u$ specified in these nodes, we can write following matrix expression

$$
\mathbf{u}=\mathbf{F} \boldsymbol{\alpha}
$$

where 


$$
\begin{gathered}
\mathbf{u}=\left[\begin{array}{lll}
u\left(\mathbf{x}_{1}\right) & \ldots & u\left(\mathbf{x}_{N_{s}}\right)
\end{array}\right]^{T} \\
\mathbf{F}=\left[\begin{array}{ccc}
F\left(\left\|\mathbf{x}_{1}-\mathbf{x}_{1}\right\|\right) & \ldots & F\left(\left\|\mathbf{x}_{1}-\mathbf{x}_{N S}\right\|\right) \\
\vdots & \ddots & \vdots \\
F\left(\left\|\mathbf{x}_{N S}-\mathbf{x}_{1}\right\|\right) & \cdots & F\left(\left\|\mathbf{x}_{N S}-\mathbf{x}_{N S}\right\|\right)
\end{array}\right] \\
\boldsymbol{\alpha}=\left[\begin{array}{lll}
\alpha_{1} & \cdots & \alpha_{N S}
\end{array}\right]^{T}
\end{gathered}
$$

Since $\mathbf{F}$ is invertible, we will obtain the unknown weighting coefficients as

$$
\boldsymbol{\alpha}=\mathbf{F}^{-1} \mathbf{u}
$$

Now, for operators concerned in solving convection-diffusion-reaction equations, we firstly consider the operator with highest order, the Laplace operator $\nabla^{2}$, and let

$$
\nabla^{2} u\left(\mathbf{x}_{i}\right)=\sum_{i=1}^{N S} \alpha_{i} f\left(\left\|\mathbf{x}-\mathbf{x}_{i}\right\|\right)=\mathbf{f} \boldsymbol{\alpha}
$$

where $f$ is the radial basis function with the essential condition as $f=\nabla^{2} F$. Replacing $\boldsymbol{\alpha}$ with Eq.(6) we get

$$
\nabla^{2} u\left(\mathbf{x}_{i}\right)=\mathbf{f F}^{-1} \mathbf{u}=\boldsymbol{\varphi} \mathbf{u}
$$

where $\boldsymbol{\varphi}$ is the vector of shape functions Laplacian. We apply similar procedures to the gradient operator $\nabla$ and we get

$$
\nabla_{j} u\left(\mathbf{x}_{i}\right)=\sum_{i=1}^{N S} \alpha_{i} \frac{\partial F\left(\left\|\mathbf{x}-\mathbf{x}_{i}\right\|\right)}{\partial x_{j}}=\mathbf{G}_{, j} \boldsymbol{\alpha}=\mathbf{G}_{, j} \mathbf{F}^{-1} \mathbf{u}=\boldsymbol{\gamma}_{j} \mathbf{u}
$$

where $\gamma_{j}$ is the vector of shape function gradients and subscript $j$ represents the coordinate of the gradient. The processed operators may now be applied directly into numerical implementations, which discretize the governing equations and the essential conditions into formulation of linear systems. All boundary conditions in this paper have also been processed using the LMAPS.

\section{LMAPS implementation of convection-diffusion equations}

\subsection{Integrated multiquadrics radial basis functions}

The trial function basis used in the LMAPS incorporates multiquadrics RBF, but different approach than the standard meshless collocation methods is adopted. In the case of LMAPS the MQ-RBF [8] represents the form of basis function after differentiation using highest order operator. To obtain the basis function, MQ-RBF needs to be integrated. In this article the two-dimensional integrated multiquadrics radial basis function (integrated MQ-RBF) is used as the basis function $F$. The MQ-RBF 


$$
f(r)=\sqrt{r^{2}+c^{2}}
$$

and the integrated MQ-RBFs [8]

$$
F(r)=\frac{1}{9}\left(r^{2}+4 c^{2}\right) \sqrt{r^{2}+c^{2}}-\frac{1}{3} c^{3} \ln \left(\sqrt{r^{2}+c^{2}}+c\right)
$$

where $r$ depicts the distance between the collocation points and $c$ is a shape parameter.

\subsection{Handling derivative boundary conditions}

Strong-form methods can produce accurate results for partial differential equations, when the boundary conditions are all of Dirichlet type. If there is any derivative boundary condition, the accuracy of the solution deteriorates drastically, and the solution can be unstable; small changes in the setup of the problem can lead to a large change in the solution.

The discretized system equation behaves like an ill-posed problem in which errors introduced into the system are magnified in the output. A number of strategies can be used to impose the derivative boundary conditions in the strong form methods. The method using fictitious points is used in this article to enforce the pressure gradient on the boundary. Along the derivative boundaries, a set of fictitious points is added outside the problem domain. In this case, two sets of equations are established at each derivative boundary node, one for the derivative boundary condition, and the other for the governing equation.

\subsection{Handling derivative boundary conditions}

In this section, the numerical procedures for the approximation of the convectiondiffusion-reaction equations via the LMAPS will be explained. The solved equation is assumed in following form:

$$
D \frac{d^{2} u}{d x^{2}}-V_{x} \frac{d u}{d x}-k u=0
$$

Using the LMAPS principles the equation (12) is discretized as follows

$$
\begin{aligned}
& D \boldsymbol{\varphi} \mathbf{u}-V_{x} \gamma_{x} \mathbf{u}-\boldsymbol{\psi} \mathbf{u}=0 \\
& {\left[D \boldsymbol{\varphi}-V_{x} \gamma_{x}-\boldsymbol{\psi}\right] \mathbf{u}=0}
\end{aligned}
$$

Which results in classical linear system of equations $\mathbf{A x}=\mathbf{b}$.

\section{Numerical example}

The example used is a convection-diffusion-reaction equation with variable velocity field and reaction term as given in subchapter 3.3. A rectangular domain with length $L=1 \mathrm{~m}$ and width $W=0.2 \mathrm{~m}$ is considered. The following BCs were applied: 


$$
u(0, y)=U_{0}=300 ; u(L, y)=U_{1}=10 ;\left.\frac{\partial u}{\partial y}\right|_{y=W / 2}=\left.\frac{\partial u}{\partial y}\right|_{y=-W / 2}=0
$$

The velocity field is defined as

$$
V_{x}=\ln \frac{U_{1}}{U_{0}}+k\left(x-\frac{1}{2}\right) ; V_{y}=0
$$

The analytical solution of the above problem [9] for $L=1 \mathrm{~m}$ and $D=1 \mathrm{~m}^{2} \mathrm{~s}^{-1}$ is given by

$$
u(x)=U_{0} \exp \left[\frac{k}{2} x^{2}+\left(n \frac{U_{1}}{U_{0}}-\frac{k}{2}\right) x\right]
$$

Though the analytical solution is $1 \mathrm{D}$, the solution is a $2 \mathrm{D}$ one since the code needs to accurately predict that in the $y$-direction the potential is constant for a given $x$, i.e. $u(x, y)=u(x)$, and also that the flux is zero in the $y$-direction. This example is particularly challenging to solve because the velocity changes direction inside the domain. For example, for $k=10$ and 40 it is in opposite direction to the $x$-axis for $x$ smaller than 0.15988 and 0.41497 , respectively, and is in the direction of the $x$-axis for $x$ larger than 0.15988 and 0.41497 , respectively. The conservation of mass forces the solution to have high gradients in the part of the domain close to $x=0$, in order for the diffusion to counterbalance the convection in the opposite direction to the $x$-axis. The maximum Peclet numbers for the examples are $P e_{\max }=8.4$, for $k=10$, and $P e_{\max }=23.4$ for $k=40$. This example has been used as a benchmark in a number of previous studies $[9,10,11]$.
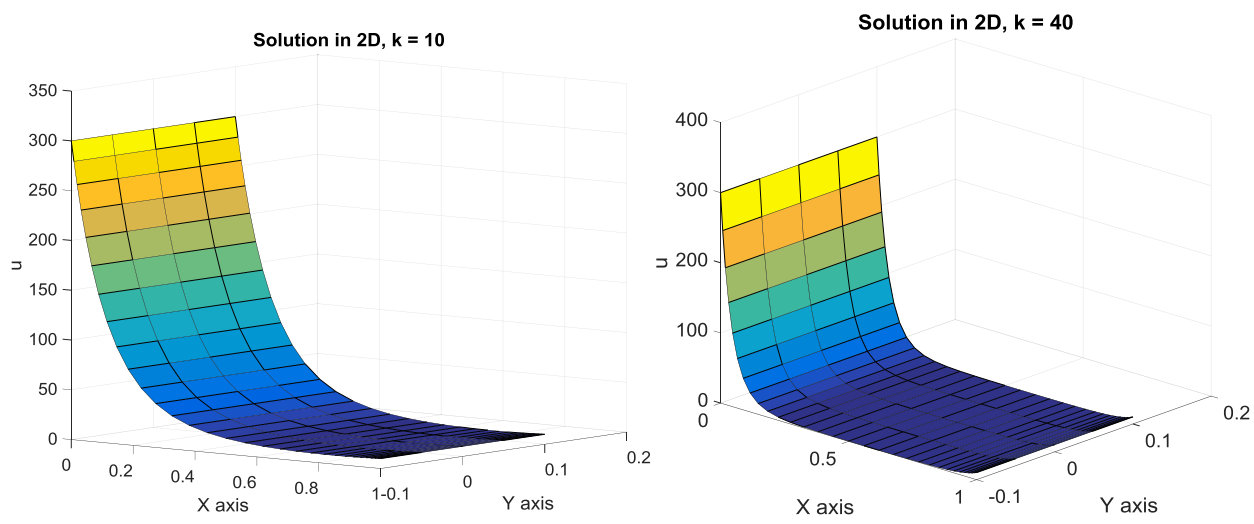

Fig. 2. Numerical results in visualised in $3 \mathrm{D}$, obtained with the LMAPS $(k=10$ and $k=40)$

Two values of $k$ were considered: 10 and 40 . The analytical results and the numerical results obtained by using the LMAPS for the case of $k=10$ and $k=40$ are shown in Fig. 2. Fig. 3 shows that the results obtained from the meshless approach agree well with the analytical results. 

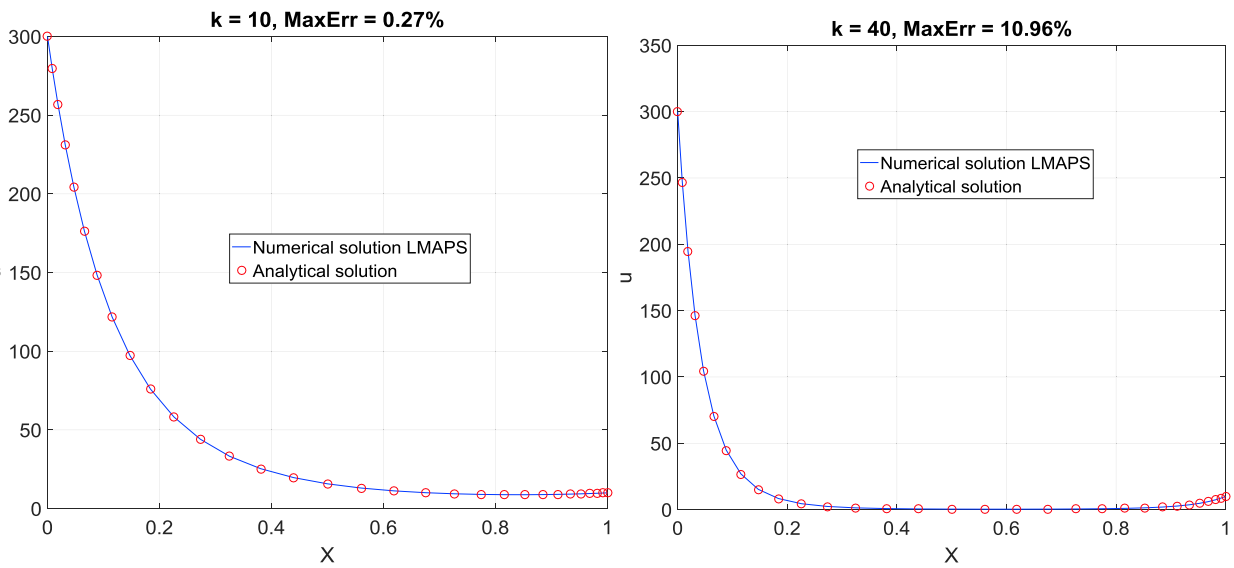

Fig. 3. Comparison of the results obtained with the LMAPS with the analytical solution $(k=10$ and $k=40)$

In Table 1 the value of potentials obtained using the LMAPS are compared with the analytical solution (16), the results obtained using the DRM-MD (dual reciprocity-multiple domain) [9], RBIEM (radial boundary integral equation method) [9] and the results obtained using Galerkin FEM (finite element method). In this case the main comparison of the accuracy of the method is considered to be with the RBIEM approach. The relative errors for the potential obtained using the four numerical methods are given in the same table. The relative error is calculated by the following formula:

$$
e_{r}=\frac{\left|u_{i}^{n}-u_{i}\right|}{u_{i}} 100 \%
$$

where $u_{i}^{n}$ is the analytical solution for $u$ and $u_{i}$ are potential values at node $i$ obtained by the numerical method. It can be seen that the LMAPS produced results with accuracy comparable with RBIEM.

Table 1. Comparison of the results for potential obtained using the MLPG compared with the analytical solution and results of RBIEM, DRM-MD and Galerkin FEM for a regular scheme of 185 computational nodes (case $\mathrm{k}=40$ ).

\begin{tabular}{|c|c|c|c|c|c|c|c|c|c|}
\hline $\mathrm{x}$ & $\begin{array}{c}\text { Analytical } \\
\text { solution }\end{array}$ & LMAPS & RBIEM & DRM-MD & $\begin{array}{c}\text { Galerkin } \\
\text { FEM } \\
\end{array}$ & $\begin{array}{c}\text { Error } \\
(\%) \\
\text { LMAPS }\end{array}$ & $\begin{array}{c}\text { Error } \\
(\%) \\
\text { RBIEM }\end{array}$ & $\begin{array}{c}\text { Error } \\
(\%) \\
\text { DRM- } \\
\text { MD } \\
\end{array}$ & $\begin{array}{c}\text { Error } \\
(\%) \\
\text { Galerkin } \\
\text { FEM } \\
\end{array}$ \\
\hline 0.00 & 300.00000 & 300.00000 & 300.00000 & 300.00000 & 300.00000 & 0.00000 & 0.00000 & 0.00000 & 0.00000 \\
\hline 0.02 & 189.38055 & 190.53640 & 179.04666 & 187.61755 & 187.68159 & 0.60662 & 5.45668 & 0.93093 & 0.90054 \\
\hline 0.04 & 121.47817 & 124.03792 & 118.35698 & 118.98971 & 119.41530 & 2.06368 & 2.56934 & 2.04848 & 1.69814 \\
\hline 0.07 & 64.30894 & 66.02516 & 63.18108 & 62.42024 & 61.66200 & 2.59934 & 1.75382 & 2.93691 & 4.11598 \\
\hline 0.10 & 35.29222 & 37.19934 & 35.16106 & 34.32921 & 33.14000 & 5.12675 & 0.37164 & 2.72869 & 6.09828 \\
\hline 0.14 & 16.77039 & 17.86164 & 17.05505 & 16.68902 & 15.10380 & 6.10947 & 1.69740 & 0.48519 & 9.93769 \\
\hline 0.19 & 7.23952 & 7.75684 & 7.47060 & 7.60905 & 6.12290 & 6.66924 & 3.19192 & 5.10446 & 15.42395 \\
\hline 0.25 & 3.01465 & 3.38576 & 3.14630 & 3.44925 & 2.36960 & 10.96102 & 4.36701 & 14.41637 & 21.39718 \\
\hline 0.32 & 1.30135 & 1.41994 & 1.34937 & 1.63377 & 0.96120 & 8.35223 & 3.69001 & 25.54530 & 26.13824 \\
\hline 0.40 & 0.63337 & 0.69743 & 0.66253 & 0.85084 & 0.45580 & 9.18422 & 4.60394 & 34.33515 & 28.03575 \\
\hline 0.56 & 0.32339 & 0.33747 & 0.34728 & 0.43739 & 0.25190 & 4.17010 & 7.38737 & 35.25292 & 22.10643 \\
\hline 0.75 & 0.55040 & 0.57254 & 0.59498 & 0.69063 & 0.48900 & 3.86825 & 8.09956 & 25.47853 & 11.15552 \\
\hline 0.85 & 1.30052 & 1.31763 & 1.32975 & 1.56886 & 1.21380 & 1.29873 & 2.24756 & 20.63368 & 6.66810 \\
\hline 0.93 & 3.45102 & 3.47837 & 3.28041 & 3.92384 & 3.33370 & 0.78630 & 4.94376 & 13.70113 & 3.39957 \\
\hline 1.00 & 10.00000 & 10.00000 & 10.00000 & 10.00000 & 10.00000 & 0.00000 & 0.00000 & 0.00000 & 0.00000 \\
\hline
\end{tabular}




\section{Conclusions}

A meshless method based on the strong formulation and combined with the local support domain approach has been proposed for solving the convection-diffusion equation. The strong form of convection-diffusion equations is solved at each node. Integrated radial basis (RBF) function interpolation is applied in order to obtain the values of the field variable.

The current approach does not require any integration over the domain or boundary of the model but the application of Neuman type boundary condition requires use of "ghost" points in order to apply the governing equation together with boundary operator of the boundary condition. The accuracy of the method has been compared with the accuracy of the RBIEM, DRM-MD with overlapping sub-domains and the FEM. In all cases the current approach has shown equal to superior accuracy to the formulation which uses a mesh. Though the method has been applied to $2 \mathrm{D}$ problems, extension of the approach to $3 \mathrm{D}$ problems is straightforward.

This contribution is the result of the project implementation: "Formulation of the new progressive numerical approaches for debris flow simulation" No. 1/0716/17 supported by the Scientific Grant Agency of Slovak Republic (VEGA)..

\section{References}

1. K. Kovářík, J. Mužík, M.S. Mahmood, Engineering Analysis with Boundary Elements, 36, 738-743, (2012)

2. G.R. Liu, M.B. Liu, Smoothed Particle Hydrodynamics: a meshfree particle method (World Scientific, Singapor, 2003)

3. E.J. Kansa, Computers and Mathematics with Application, 19, 127-145, (1990)

4. S.N. Atluri, T. Zhu, Computational Mechanics, 22, 117-127, (1998)

5. C.S. Chen, C.M. Fan, P.H. Wen, Numerical Methods for Partial Differential Equations, 28, 506-522, (2012)

6. C.S. Chen, M.A. Golberg, M. Ganesh, A.H.-D. Computers and Mathematics with Application, 43, 359-378, (2002)

7. E.J. Sellountos, A. Sequeira, Comput Mech, 41, 617, (2008)

8. J. Mužík, L. Kais, R. Bulko, Communications - Scientific Letters of the University of Zilina, 18, 77-82, (2016)

9. V. Popov, T.T. Bui, Engineering Analysis with Boundary Elements, 34, 680-689, (2010)

10. V. Popov, H. Power, International Journal for Numerical Methods in Engineering, 44, 327-353, (1999)

11. V. Popov, H. Power, Engineering Analysis with Boundary Elements, 23, 175-88, (1999) 